\title{
Coexistence of a charge density wave and superconductivity in the cluster compound
}

\section{$\mathrm{K}_{2} \mathrm{Mo15Se}_{19}$}

Christophe Candolfi ${ }^{1, *}$, Martin Míšek ${ }^{2}$, Patrick Gougeon ${ }^{4, *}$, Rabih Al Rahal Al Orabi ${ }^{3,4}$, Philippe Gall ${ }^{4}$, Régis Gautier ${ }^{4}$, Sylvie Migot ${ }^{1}$, Jaafar Ghanbaja ${ }^{1}$, Jiř́ Kaštil ${ }^{2}$, Petr Levinský², Jiř́i Hejtmánek ${ }^{2}$, Anne Dauscher ${ }^{1}$, Bernard Malaman ${ }^{1}$, Bertrand Lenoir $^{1}$

${ }^{1}$ Institut Jean Lamour, UMR 7198 CNRS - Université de Lorraine, Campus ARTEM, 2 allée André Guinier, BP 50840, 54011 Nancy, France

${ }^{2}$ Institute of Physics, Czech Academy of Sciences, Cukrovarnická 10, 162 00, Praha 6, Czech Republic

${ }^{3}$ Solvay, Design and Development of Functional Materials Department, Axel'One, 87 avenue des Frères Perret, 69192 Saint Fons, Cedex, France

${ }^{4}$ Univ Rennes, INSA-ENSCR, CNRS, ISCR - UMR 6226, 35000 Rennes, France

\begin{abstract}
\end{abstract}
The competition between charge density wave (CDW) and superconductivity is a central theme in condensed-matter physics with ramifications to correlated electron systems and hightemperature superconductivity. While the emergence of superconductivity is often observed upon suppressing the CDW transition by tuning the chemical composition or the external pressure, their coexistence has been reported in only a handful of materials, with the cuprates being the most prominent example. Here, we demonstrate that both cooperative electronic phenomena coexist in the cluster compound $\mathrm{K}_{2} \mathrm{Mo}_{15} \mathrm{Se}_{19}$. The CDW transition sets in at $T_{\mathrm{CDW}}$ $=114 \mathrm{~K}$, accompanied by a commensurate periodic modulation of the crystal lattice along the 
$c$ axis evidenced by electron diffraction. Bulk type-II superconductivity develops upon further cooling below $T_{\mathrm{c}}=2.8 \mathrm{~K}$. The presence of similar signatures of $\mathrm{CDW}$ ordering in other $A_{2} \mathrm{Mo}_{15} \mathrm{Se}_{19}$ compounds show that this electronic instability may be ubiquitous in these compounds, providing a novel family where the interplay between CDW and superconductivity may be systematically investigated.

\section{INTRODUCTION}

Periodic modulations of the conduction electrons, referred to as charge density wave (CDW), are often observed in low-dimensional systems such as quasi-1D chain-like and 2D layered materials [1-3]. While phonon-mediated superconductivity is favored by a high density of states at the Fermi level $N\left(E_{F}\right)$, the coupling between the electronic and lattice degrees of freedom gaps $N\left(E_{F}\right)$ through the appearance of a different structural periodicity at the Fermi wave vector below the transition temperature $T_{\mathrm{CDW}}$. Because of these two antagonistic characteristics, CDW and superconductivity are widely thought of as being two competing electronic orders $[3,4]$. In many CDW systems such as the canonical layered dichalcogenides [1,5], suppressing the long-range CDW order through intercalation, substitution or external pressure often leads to the emergence of superconductivity [6-9]. In some specific cases, these tuning parameters continuously drive the transition temperature $T_{\mathrm{CDW}}$ towards $T=0 \mathrm{~K}$, marking the onset of a structural quantum critical point (SQCP) near which superconductivity also develops [10-13]. Because the appearance of unconventional superconductivity is often furnished by destroying such an ordered phase, the study of the interplay between these two electronic phenomena remains a central theme in condensed matter physics, reinforced by the recent discovery of CDW order in high-temperature cuprate superconductors [14-17]. Despite the discovery of many CDW materials to date, only a handful of them shows a coexistence of 
CDW and superconductivity under ambient pressure or without manipulation of their chemical composition [18-26]. In addition to Heusler phases such as $\mathrm{LuPt}_{2} \mathrm{In}$ [10] or the ternary rareearth dicarbides $R \mathrm{NiC}_{2}(R=$ rare earth) [27-30], the ternary stannides crystallizing with a $\mathrm{Yb}_{3} \mathrm{Rh}_{4} \mathrm{Sn}_{13}$-type structure have recently attracted attention due to the interplay between CDW and superconductivity and the possibility to induce a SQCP [11,13,31-33]. Identifying novel systems supporting both phenomena would provide interesting platforms to systematically investigate their interplay via various probes, such as external pressure or intentionally introducing weak disorder by electron irradiation or substitutions [34,35], and possibly induce SQCP through chemical substitutions. Furthermore, the coexistence of both electronic orders in a single material may open the possibility to observe the Higgs mode in Raman spectra below the superconducting critical temperature $T_{\mathrm{c}}[36-39]$.

Mo-based cluster compounds are a large class of materials that were recently shown to display interesting thermoelectric properties [40-50]. Their crystal structures are built up by $\mathrm{Mo}_{3 n} X_{3 n+2}(X=\mathrm{S}, \mathrm{Se}$ or Te$)$ clusters units in which the nuclearity of the Mo3n clusters can vary from Mo3 up to Mo36 [51-53]. Many of them show superconductivity with some rare examples of emergence of superconductivity within the antiferromagnetic state or of ferromagnetic reentrant superconductivity (the so-called Jaccarino-Peter effect) in the Chevrel phases $L n \mathrm{Mo}_{6} \mathrm{~S}_{8}(L n=\mathrm{Tb}, \mathrm{Dy}, \mathrm{Er})$ and $\mathrm{HoMo6}_{6} \mathrm{~S}_{8}$, respectively [54-57]. The low dimensionality of the crystal structure of some of these compounds make them prone to various structural or electronic instabilities and hence, a fertile area for searching novel materials experiencing symmetry-breaking density wave transitions.

Among the numerous families of Mo-based cluster compounds known to date, the compounds $A_{2} \mathrm{Mo}_{15} \mathrm{Se}_{19}(A=\mathrm{Li}, \mathrm{K}, \mathrm{Na}, \mathrm{Rb}, \mathrm{Ba}, \mathrm{Sn}, \mathrm{In}, \mathrm{Pb}$ or $\mathrm{Tl})$ have been shown to become superconducting with a critical temperature $T_{\mathrm{c}}$ of up to $2.8 \mathrm{~K}$ that depends on the nature of the $A$ atoms $[58,59]$. Here, we consider the $\mathrm{K}_{2} \mathrm{Mo}_{15} \mathrm{Se}_{19}$ compound and demonstrate that the 
previously reported superconducting state emerging at $T_{\mathrm{c}}=2.8 \mathrm{~K}$ [59] coexists with a CDW transition that sets in upon cooling below $T_{\mathrm{CDW}}=114 \mathrm{~K}$. The CDW transition is accompanied by a weak commensurate modulation that doubles the $c$ axis of the room-temperature hexagonal crystal lattice shown in Figure 1. Preliminary low-temperature measurements performed on other $A_{2} \mathrm{Mo}_{15} \mathrm{Se}_{19}$ compounds $(A=\mathrm{In}, \mathrm{Rb}, \mathrm{Tl})$ further indicate that the coexistence of superconductivity and CDW may be commonly observed in this family. Due to the large variety of $A$ atoms that can be accommodated by this crystal structure, the $A_{2} \mathrm{Mo}_{15} \mathrm{Se}_{19}$ family is a novel intriguing system in which the interplay between these two electronic orders can be systematically investigated.

\section{EXPERIMENTAL AND COMPUTATIONAL DETAILS}

All the elements and precursors were handled in an argon-filled glovebox to avoid contamination by oxygen and moisture. Prior to use, Mo powders were reduced under $\mathrm{H}_{2}$ flowing gas at $1000^{\circ} \mathrm{C}$ during $10 \mathrm{~h}$ to eliminate possible traces of oxygen. $\mathrm{MoSe}_{2}, \mathrm{~K}_{2} \mathrm{Mo}_{6} \mathrm{Se}_{6}$ and Mo were used in powder form as starting materials for the solid-state syntheses. $\mathrm{MoSe}_{2}$ was prepared by direct reaction of stoichiometric quantities of the elements in an evacuated, sealed silica tube, heated to $700^{\circ} \mathrm{C}$ and maintained at this temperature for two days. $\mathrm{K}_{2} \mathrm{Mo}_{6} \mathrm{Se}_{6}$ was synthesized by a two-step process. As a first step, $\operatorname{In}_{2} \mathrm{Mo}_{6} \mathrm{Se}_{6}$ was prepared from a stoichiometric mixture of $\mathrm{InSe}, \mathrm{MoSe}_{2}$ and $\mathrm{Mo}$ at $1000^{\circ} \mathrm{C}$ for $36 \mathrm{~h}$ in an evacuated, sealed silica tube. The second step consisted in an ion-exchange reaction of $\operatorname{In}_{2} \mathrm{Mo}_{6} \mathrm{Se}_{6}$ with $\mathrm{KCl}$ at $800^{\circ} \mathrm{C}$. Powders of $\mathrm{In}_{2} \mathrm{Mo}_{6} \mathrm{Se}_{6}$ and $\mathrm{KCl}$ were mixed in a ratio 1:2.5 before being cold pressed into cylindrical pellets. The pellets were subsequently sealed under vacuum in a long silica tube. The end of the tube containing the pellet was placed in a furnace, leaving about $5 \mathrm{~cm}$ of the other end out of the furnace. The furnace was heated at $800^{\circ} \mathrm{C}$ for $48 \mathrm{~h}$. After reaction, white 
crystals of $\mathrm{InCl}$ were observed at the cold end of the tube. All starting reagents were determined to be monophasic according to their powder X-ray diffraction patterns. Phase-pure polycrystalline samples of $\mathrm{K}_{2} \mathrm{Mo}_{15} \mathrm{Se}_{19}$ were finally prepared from stoichiometric quantities of $\mathrm{K}_{2} \mathrm{Mo}_{6} \mathrm{Se}_{6}$, Mo and $\mathrm{MoSe}_{2}$ heated at $1300^{\circ} \mathrm{C}$ during $40 \mathrm{~h}$ in a Mo crucible, sealed under a low pressure of argon using an arc-welding system. The obtained ingot was ground into fine powders that were subsequently consolidated by hot uniaxial pressing under vacuum (about $10^{-}$ $\left.{ }^{2} \mathrm{mbar}\right)$. To this end, the powders were introduced into a graphite die $(\varnothing 12 \mathrm{~mm})$, previously coated with boron nitride. The consolidation process was realized at $1300^{\circ} \mathrm{C}$ under a uniaxial pressure of $85 \mathrm{MPa}$ with a dwell time of $2 \mathrm{~h}$. The density of the consolidated cylindrical pellet, determined from the weight and geometric dimensions, was calculated to be around $98 \%$ of the theoretical density from crystallographic data.

Single crystals of $\mathrm{K}_{2} \mathrm{Mo}_{15} \mathrm{Se}_{19}$ were grown by heating a polycrystalline sample at $1750^{\circ} \mathrm{C}$ for $1 \mathrm{~h}$, cooled to $1000^{\circ} \mathrm{C}$ at $100^{\circ} \mathrm{C} \mathrm{h}^{-1}$ and finally furnace-cooled to room temperature. Cuboidshaped single crystals of typical dimensions of $\sim 300 \times 200 \times 200 \mu \mathrm{m}^{3}$ with grey metallic luster could be isolated.

The purity of the polycrystalline sample was checked by room temperature powder x-ray diffraction (PXRD) using a Bruker D8 Advance $\left(\mathrm{CuK \alpha} \alpha_{1}\right.$ radiation, $\left.\lambda=1.54056 \AA\right)$ diffractometer. Additional PXRD measurements were performed at $100 \mathrm{~K}$ using an Xpert Pro MPD diffractometer $\left(\mathrm{CuK} \alpha_{1}\right.$ radiation, $\lambda=1.54056 \AA$ and $\mathrm{CuK} \alpha_{2}$ radiation, $\lambda=1.54439 \AA$, ratio $\left.\mathrm{K} \alpha_{2} / \mathrm{K} \alpha_{1}=0.5\right)$.

The crystal structure of single crystals was verified by XRD at $300 \mathrm{~K}$ and further measured down to $100 \mathrm{~K}$ on a Bruker APEX-II diffractometer equipped with a CCD detector using MoK $\alpha$ radiation $(\lambda=0.71073 \AA)$. The structure solution was determined by direct methods and refined by full-matrix least-squares techniques with the Sir97 and SHELX-2013 software, respectively, implemented through the WinGX program package [60]. Details of 
structure refinements and refined crystallographic parameters are given in Tables S1 and S2, respectively, in Supplemental Material [61].

For transmission electron microscopy experiments, a thin slice was prepared from a grown single crystal of $\mathrm{K}_{2} \mathrm{Mo}_{15} \mathrm{Se}_{19}$ by the dual focused ion beam (FIB) - scanning electron microscope system using the in situ lift-out technique. At $300 \mathrm{~K}$, scanning TEM (STEM) measurements in high-angle annular dark-field (HAADF), annular bright-field (ABF) and high resolution (HRTEM) modes were carried out on a JEOL ARM 200F-cold FEG microscope operating at $200 \mathrm{keV}$. This system is equipped with a spherical aberration (Cs) probe and image correctors. In situ cooling TEM experiments were performed using a double-tilt liquidnitrogen-cooling TEM sample holder (Gatan Inc., USA), which can reach temperatures of about $100 \mathrm{~K}$. The sample was slowly cooled down to the lowest temperature and the experiments were conducted after a significant waiting time in the absence of the electron beam to achieve thermal equilibrium. Selected area electron diffraction (SAED) and HRTEM images were collected at about $100 \mathrm{~K}$ along various zone axes.

Measurements of the transport properties were performed on samples cut from the consolidated polycrystalline pellet using a diamond-wire saw. At low temperatures $(2-300 \mathrm{~K})$, a Quantum Design Physical Property Measurement System (PPMS) was used to measure simultaneously the temperature dependence of the electrical resistivity, thermopower and thermal conductivity with the thermal transport option (TTO). For this measurement, four copper leads were mounted on the samples using a silver-containing conductive epoxy.

Specific heat measurements under zero magnetic field were performed in the temperature range $0.35-4 \mathrm{~K}$ using the ${ }^{3} \mathrm{He}$ option of the PPMS system. A small polycrystalline piece of approximately $20 \mathrm{mg}$ was glued with a tiny amount of Apiezon $\mathrm{N}$ grease to ensure a good thermal contact between the sample and the platform of the specific heat puck. Further measurements with magnetic field sweeps from 0 up to $3 \mathrm{~T}$ were performed. For all 
measurements, the contribution of the grease has been systematically measured under the same magnetic fields prior to gluing the sample.

Magnetic property measurements were carried out between 2 and $300 \mathrm{~K}$ using a Magnetic Property Measurement System (MPMS) Superconducting Quantum Interference Device (SQUID) magnetometer. The magnetic susceptibility $\chi$ was determined from the slope of the magnetization curves, $\chi=\partial M / \partial \mu_{0} H$ for magnetic fields $\mu_{0} H \rightarrow 0$, measured as a function of temperature. These measurements were performed on a piece of the polycrystalline bar-shaped sample used for TTO measurements of approximately $90 \mathrm{mg}$. The sample was loaded in a gelatin capsule which was mounted in a plastic straw. Further $M\left(\mu_{0} H\right)$ curves were measured below $3 \mathrm{~K}$ to determine the lower and upper critical fields $H_{c 1}$ and $H_{c 2}$, respectively. Zerofield-cooled (ZFC) susceptibility data were collected below $10 \mathrm{~K}$ with an applied field of 10 Oe. The ZFC data have been corrected for a demagnetization factor $N=0.456$ estimated using the formula derived in Ref. 62 for a rectangular cuboid shape.

Density functional theory (DFT) calculations were carried out using the WIEN2k program package [63]. The generalized gradient approximation (GGA) exchange-correlation functional within the parameterization of Perdew, Burke and Ernzehof (PBE) was used for calculations [64]. A plane-wave cutoff corresponding to $R_{M T} K_{\max }=7$ was used with the radial wave-functions inside the nonoverlapping muffin-tin spheres surrounding the atoms expanding up to $l_{\max }=12$. The charge density was Fourier expanded up to the reciprocal vector $G_{\max }=$ $14 \AA^{-1}$. Total energy convergence was achieved with respect to the Brillouin zone (BZ) integration using a mesh of $500 k$-points. We used $3000 k$-points in the $\mathrm{BZ}$ to compute the electronic band structure and Fermi surface. Views of the Fermi surface were obtained using the software XCrySDen [65]. The dispersion curves were shifted so that the Fermi level $E_{F}$ lies at $0 \mathrm{eV}$. Because spin-orbit coupling (SOC) hardly modifies the electronic band structure of $\mathrm{K}_{2} \mathrm{Mo}_{15} \mathrm{Se}_{19}$ (see Figure S1 in Supplemental Material [61]), SOC was not taken into account. 


\section{RESULTS}

A first clear experimental evidence for a CDW transition in $\mathrm{K}_{2} \mathrm{Mo}_{15} \mathrm{Se}_{19}$ is provided by the temperature dependence of the electrical resistivity $\rho(T)$ shown in Figure 2a. Upon cooling, a metallic-like dependence prevails down to about $115 \mathrm{~K}$ where an upturn in $\rho(T)$ can be observed, followed by a broad maximum. These two characteristics are typical signatures of a CDW transition with an onset temperature of $T_{\mathrm{CDW}}=114 \mathrm{~K}[10]$. Below the broad maximum, $\rho(T)$ decreases upon further cooling to $3 \mathrm{~K}$.

The anomaly seen in $\rho(T)$ leads to corresponding signatures in the magnetic susceptibility $\chi(T)$, specific heat $C_{p}(T)$ and thermopower $\alpha(T)$ (Figures $2 \mathrm{~b}, 2 \mathrm{c}$ and $2 \mathrm{~d}$ ). Above $T_{\mathrm{CDW}}, \chi(T)$ is practically temperature-independent as expected in a non-magnetic metal. The positive values measured are due to the Pauli susceptibility $\chi_{P}$ of the conduction electrons that outweighs the diamagnetic $\chi_{d i a}$ and Van Vleck $\chi_{V V}$ contributions. A drop in $\chi(T)$ is observed as the temperature decreases and passes through $T_{\mathrm{CDW}}$. The decrease in the $\chi$ values is consistent with a gap opening in the electronic density of states at the Fermi level. However, the fact that the $\chi(T)$ values remain positives below $T_{\mathrm{CDW}}$ implies that $\chi_{P}$ is still the main contribution indicating only a partial gapping. These conclusions are further corroborated by the evolution of the thermopower (Figure 2d) which represents a sensitive probe of changes in the Fermi surface. The linear variation upon cooling, characteristic of a diffusive regime in a metallic compound, shows a sharp increase at $T_{\mathrm{CDW}}$, peaks near $70 \mathrm{~K}$ before decreasing down to the lowest temperature measured. The CDW transition is not accompanied by a change in the dominant carrier type which remains hole-like over the entire temperature range. The slight but visible increase in $\alpha$ below $T_{\mathrm{CDW}}$ is consistent with a decrease in the hole concentration as a result of the partial gapping at $N\left(\varepsilon_{F}\right)$. 
The anomalies seen in the magnetic and transport coefficients are not marked by a sudden, step-like variation suggesting a second-order phase transition in the present case. Although unusual for this type of transition, which is usually first-order, the second-order nature of the present CDW is not unprecedented and has been observed, for instance, in the ternary $\mathrm{Ce}_{3} \mathrm{Rh}_{4} \mathrm{Sn}_{13}$ [33] or in the $\mathrm{Lu}\left(\mathrm{Pt}_{1-x} \mathrm{Pd}_{x}\right)_{2} \mathrm{In}$ alloys for which similar temperature dependences of $\rho$ and $\chi$ have been reported [10]. Measurements of $\chi$ performed on cooling and warming across $T_{\text {CDW }}$ did not reveal any hysteretic behavior to within experimental uncertainty, further suggestive of a second-order transition (Figure S2 in Supplemental Material [61]).

One important characteristic of the present CDW transition is the absence of a welldefined peak in the specific heat at $T_{\mathrm{CDW}}$, should it be measured upon cooling or warming (Figure 2c). In the present case, the $C_{p}$ values plateau from 114 down to $110 \mathrm{~K}$, the temperature at which $C_{p}(T)$ decreases again upon cooling. In contrast, most of the CDW systems studied so far displays a large specific heat jump at $T_{\mathrm{CDW}}$ associated with a non-negligible entropy variation across the transition $[10,66,67]$. Such a commonly behavior has been observed for both first-order and second-order CDW transitions and is therefore rather tied to the periodic lattice distortion that accompanies the CDW $[10,66,67]$. The absence of significant anomaly in $C_{p}(T)$ suggests that the $\mathrm{CDW}$ in $\mathrm{K}_{2} \mathrm{Mo}_{15} \mathrm{Se}_{19}$ is not strongly coupled synonymous with a small amplitude of the lattice distortion.

The presence of a modification of the crystal lattice accompanying the CDW transition in $\mathrm{K}_{2} \mathrm{Mo}_{15} \mathrm{Se}_{19}$ has been directly observed by electron diffraction (Figure 3). While the roomtemperature STEM images (Figures 3a and 3b) are consistent with the hexagonal crystal structure of $\mathrm{K}_{2} \mathrm{Mo}_{15} \mathrm{Se}_{19}$, electron diffraction images, taken along the [10 $\left.\overline{1} 0\right]$ zone axis (equivalent to the $[01 \overline{1} 0]$ zone axis in the present case) at $300 \mathrm{~K}$ (Figure $3 \mathrm{c}$ ) and below $T_{\mathrm{CDW}}$ (Figure 3d), show that additional satellite reflections appear in addition to the main Bragg reflections. The superlattice reflections appear solely along the [0001] direction corresponding 
to a modulation of the crystal structure along the $c$ axis. The absence of modulation along the $a$ axis is further demonstrated by the image taken along the [12 $\overline{3} 0]$ zone axis (Figure S3 in Supplemental Material [61]), which does not evidence the presence of satellite reflections along the $[2 \overline{1} \overline{1} 0]$ direction. Within the accuracy of these measurements, the spacing between the main and satellite reflections leads to an integer modulation vector $\overrightarrow{q_{C D W}}=0.5 c^{*}$ indicative of a modulation commensurate with the crystal lattice. These measurements further suggest that the modulated structure retains a hexagonal symmetry, which is supported by electron diffraction images showing reflections in the first-order Laue zone (Figure S4 in Supplemental Material [61]), for which the shift observed between the zero-order and the first-order Laue zones are consistent with the symmetries of the $R \overline{3} c$ space group observed above $T_{\mathrm{CDW}}$. Thus, this lattice distortion contrasts with the rhombohedral-to-monoclinic or rhombohedral-to-triclinic distortions observed at low temperatures in some Chevrel phases, which are often of first-order nature and result in a strong hysteric behavior of the transport properties around the transition temperature [68-75]. Because the modulation of the basic unit cell of $\mathrm{K}_{2} \mathrm{Mo}_{15} \mathrm{Se}_{19}$ is weak, leaving fingerprints neither in low-temperature laboratory single-crystal X-ray diffraction nor in powder X-ray diffraction, and due to the large number of atoms in the low-temperature unit cell (432 atoms, that is, twice as high as at room temperature), further synchrotron diffraction experiments on single crystal will be necessary to ascertain the superspace group and develop a detailed structural model of the modulated structure.

Upon further cooling below $T_{\mathrm{CDW}}$, superconductivity emerges with a critical temperature $T_{\mathrm{c}}$ of $2.8 \mathrm{~K}$ as revealed by the drop of $\rho(T)$ to zero (Figure $4 \mathrm{a}$ ). This $T_{\mathrm{c}}$ value is in agreement with an early study on this compound which had reported a critical temperature of $2.45 \mathrm{~K}$ [59]. The bulk nature of the superconducting transition is further confirmed by measurements of the specific heat and magnetic susceptibility (Figures $4 \mathrm{~b}$ and $4 \mathrm{c}$, respectively). The clear meanfield-type anomaly observed in the $C_{p}(T) / T$ data at $2.6 \mathrm{~K}$, as determined by an isentropic 
construction, is consistent with the transition temperature observed in $\rho(T)$. Further evidence for a bulk phenomenon is provided by the $\chi(T)$ data which show a Meissner effect of nearly $100 \%$ indicating a full superconducting volume fraction.

A more detailed characterization of the superconducting state has been carried out by $C_{p}$ measurements under magnetic fields $\mu_{0} H$ ranging between 0 and $3 \mathrm{~T}$. As expected, applying an external magnetic field tends to shift down $T_{\mathrm{c}}$. The normal-state specific heat has been analyzed via the conventional Fermi-liquid relation $C_{p}=\gamma T+\beta T^{3}$ where $\gamma T$ represents the contribution of the free charge carriers and $\beta T^{3}$ the phonon contribution. A fit of the data plotted as $C_{p}(T) / T$ versus $T^{2}$ yields a Sommerfeld coefficient $\gamma=63 \mathrm{~mJ} \mathrm{~mol}^{-1} \mathrm{~K}^{-2}$. After subtraction of the phonon contribution, the superconducting energy gap $\Delta(0)$ was estimated from a plot on a logarithmic scale of the electronic contribution to the specific heat $C_{e l} / \gamma T_{c}$ versus $T_{c} / T$. The comparison of this curve to the Bardeen-Cooper-Schrieffer (BCS) formula predicting $C_{e l}(T)$ below $T_{c}$, $C_{e l} / \gamma T_{c}=8.5 \exp \left[-0.82 \Delta(0) / k_{B} T\right]$, where $k_{B}$ is the Boltzmann constant, yields an energy gap of $0.40 \mathrm{meV}$ corresponding to a ratio $2 \Delta(0) / k_{B} T_{c}=3.64$, that is, very close to the weakcoupling BCS value of 3.53. Further assuming a phonon-mediated pairing mechanism, the electron-phonon coupling constant $\lambda_{e-p h}$ can be inferred from the McMillan relation $T_{c}=$ $\frac{\theta_{D}}{1.45} \exp \left[\frac{-1.04\left(1+\lambda_{e-p h}\right)}{\lambda_{e-p h}-\mu^{*}\left(1+0.62 \lambda_{e-p h}\right)}\right][76]$. With the Debye temperature $\theta_{D}=183 \mathrm{~K}$ determined from the $\beta$ parameter $\left(\theta_{D}=\left(\frac{12 \pi^{4} R N}{5 \beta}\right)^{1 / 3}\right.$ with $R$ the gas constant and $N$ the number of atoms per formula unit), and assuming a Coulomb repulsion parameter $\mu^{*}$ of 0.13 as commonly used in the literature, a coupling constant $\lambda_{e-p h}$ of 0.61 is obtained, placing this compound among the weakly-to-intermediate-coupled superconductors.

Measurements of the field dependence of the magnetization $M$ performed at $2 \mathrm{~K}$ (Figure S5 in Supplemental Material [61]) give estimates of the lower $\mu_{0} H_{c 1}$ and upper $\mu_{0} H_{c 2}$ critical fields of $4 \mathrm{mT}$ and $0.6 \mathrm{~T}$, respectively. In conventional superconductors, the BCS theory 
predicts that $\mu_{0} H_{c 2}$ should exhibit a temperature dependence following the law $\mu_{0} H_{c 2} \approx$ $\mu_{0} H_{c 2}(0)\left[1-\left(T / T_{c}\right)^{2}\right]$. This expression gives an upper critical field at $0 \mathrm{~K}, \mu_{0} H_{c 2}(0)$, of approximately 2.9 T. Using a similar expression for the lower critical field yields an estimate for $\mu_{0} H_{c 1}(0)$ of $12 \mathrm{mT}$. Note that the critical field values should be taken only as estimates of the actual values due to the strongly anisotropic crystal structure of $\mathrm{K}_{2} \mathrm{Mo}_{15} \mathrm{Se}_{19}$ which likely gives rise to anisotropic critical fields. Thus, the polycrystalline nature of the sample used herein only yields average values of $\mu_{0} H_{c 1}, \mu_{0} H_{c 2}$ and $\mu_{0} H_{c 2}(0)$. Nevertheless, considering these values as reasonable approximations of the actual values, the coherence length $\xi_{\mathrm{GL}}$ and the penetration depth $\lambda_{\mathrm{GL}}$ are estimated to be $110 \AA$ and $2000 \AA$ using the two Ginzburg-Landau formulas $H_{c 2}(0)=\Phi_{0} / 2 \pi \xi_{\mathrm{GL}}^{2}$ and $H_{c 1}(0)=\frac{\Phi_{0}}{4 \pi \xi_{\mathrm{GL}}^{2}} \ln \frac{\lambda_{\mathrm{GL}}}{\xi_{\mathrm{GL}}}$, respectively, where $h$ is the Planck constant, $e$ is the elemental charge and $\Phi_{0}=h / 2 e$ is the quantum flux. From these values, the Ginzburg-Landau parameter $\kappa_{G L}=\lambda_{\mathrm{GL}} / \xi_{\mathrm{GL}}$ is equal to 18 , which further confirms the classification of $\mathrm{K}_{2} \mathrm{Mo}_{15} \mathrm{Se}_{19}$ as a type-II superconductor. The thermodynamic critical field $\mu_{0} H_{c}(0)$ can be obtained from the above-mentioned values using the relation $H_{c 1} H_{c 2}=$ $H_{C} \ln \kappa_{G L}$, yielding an estimate of $106 \mathrm{mT}$. All the superconducting parameters determined for $\mathrm{K}_{2} \mathrm{Mo}_{15} \mathrm{Se}_{19}$ are summarized in Table 1.

\section{DISCUSSION}

One important question raised by these results is the underlying physical mechanism driving the CDW ordering. While Fermi surface nesting has for long been considered as the driving force dictating the CDW wave vector $\vec{q}_{\mathrm{CDW}}$ and the corresponding lattice distortion $[77,78]$, a growing body of evidence acquired over the last years suggests that this simple scenario fails in most of the known CDW systems [78-81]. This failure led to a recentlyproposed new classification scheme of CDW with the momentum dependence of the electron- 
phonon coupling matrix playing a central role in determining the main traits of the CDW order [82].

In order to shed light on the possible origin of the $\mathrm{CDW}$ in $\mathrm{K}_{2} \mathrm{Mo}_{15} \mathrm{Se}_{19}$, we computed the electronic band structure and Fermi surface using density functional calculations (Figure 5a and Figure S6 in Supplemental Material [61]). The Fermi level, hosted by the valence bands, crosses four distinct electronic bands giving rise to a complex Fermi surface, represented in Figure 5b. The Fermi surface comprises a hole-like cylinder at the zone center $\Gamma$ surrounded by a second distorted hole-like cylinder, small electron-like elongated ellipsoids near the M and L points, and a large electron-like surface (sometimes referred to as a "monster" in the literature, see, e.g., Refs. 83 and 84) spanning most of the Brillouin zone. While 2D-like Fermi surfaces are often considered as favoring Fermi surface nesting, no significant portions of the present Fermi surface seem to be clearly connected through the experimentally observed wave vector $\vec{q}_{\text {CDW }}$, as it would be expected within a nesting scenario. Hence, these results do not seem to confirm a clear, dominant Fermi surface nesting at the observed wave vector suggesting that $\mathrm{K}_{2} \mathrm{Mo}_{15} \mathrm{Se}_{19}$ may be another example of system where the CDW is driven by $q$-dependent electron-phonon coupling. Further direct measurements of the phonon dispersions and their linewidths across $T_{\text {CDW }}$ by inelastic X-ray scattering will be important in better understanding the origin of the CDW transition in $\mathrm{K}_{2} \mathrm{Mo15}_{15} \mathrm{Se}_{19}$

The coexistence of CDW and superconductivity reported herein is likely not limited to $\mathrm{K}_{2} \mathrm{Mo}_{15} \mathrm{Se}_{19}$ but may be also observed in other $A_{2} \mathrm{Mo}_{15} \mathrm{Se}_{19}$ compounds for various $A$ atoms. The comparison of low-temperature measurements performed on the $A=\mathrm{Rb}$, In and $\mathrm{Tl}$ compounds (Figure S7 in Supplemental Material [61]) show that all of them exhibit a similar CDW signature between 100 and $150 \mathrm{~K}$ suggesting that CDW may be ubiquitous in this family of compounds. Interestingly, combined with the $T_{\mathrm{c}}$ reported for several $A_{2} \mathrm{Mo}_{15} \mathrm{Se}_{19}$ compounds (Table 2) [58], these results show that both $T_{\mathrm{CDW}}$ and $T_{\mathrm{c}}$ are not equivalent and depend on the 
nature of the $A$ atoms. Although the highest $T_{\mathrm{c}}$ values seem to be obtained for the lightest elements, the absence of superconductivity in the $\mathrm{Na}$ compound suggests a more complex dependence of $T_{\mathrm{c}}$ with the nature of the $A$ atoms. Furthermore, the presence of a more stronglycoupled CDW transition may naturally explain the absence of superconductivity in some of these compounds. Substitutions or application of high-pressure may be used as external parameters to tip the balance towards superconductivity by suppressing the CDW, thereby possibly driving the electronic system towards a QCP.

Finally, the large anisotropic thermal displacement parameters of the $\mathrm{K}$ atoms (see Table S2 in Supplemental Material [61]), and more generally of the $A$ atoms, adds another degree of complexity in this family of compounds. As observed in several cage-like compounds, large vibration amplitudes are associated with low-energy optical modes in the phonon spectrum, which are believed to be the key ingredient that limits the propagation of acoustic phonons. Meanwhile, the presence of these localized, often anharmonic, modes may also play a role in determining the superconducting transition temperature [85-88]. These so-called rattling modes may strongly couple with the conduction electrons contributing to enhance the electron-phonon coupling constant $\lambda_{e-p h}$ and hence, $T_{\mathrm{c}}$. This effect has been discussed in several families of cage-like compounds or in compounds where the rattling atoms reside in tunnels, similarly to the $A_{2} \mathrm{Mo}_{15} \mathrm{Se}_{19}$ compounds [85-88]. In these studies, the anharmonic character of the low-lying optical modes was found to correlate with $T_{\mathrm{c}}$ indicating that highly-anharmonic modes tend to reinforce superconductivity. In $A_{2} \mathrm{Mo}_{15} \mathrm{Se}_{19}$, the low-energy modes associated with the dynamics of the $A$ atoms might therefore contribute to strengthen the electron-phonon coupling, partially counterbalancing the removal of electronic states at the Fermi level upon crossing the CDW transition. Because calculations of the phonon spectra are challenging due to the large number of atoms in the unit cell above $T_{\mathrm{CDW}}$, and a fortiori below $T_{\mathrm{CDW}}$, inelastic neutron or X-ray scattering experiments would be worthwhile to determine and compare the degree of 
anharmonicity of the dynamics of the $A$ atoms in these compounds and possibly unveil a correlation between this characteristic and $T_{\mathrm{c}}$.

\section{CONCLUSION}

Low-temperature physical property measurements combined with electron diffraction revealed the coexistence of CDW and superconductivity in the Mo-based cluster compound $\mathrm{K}_{2} \mathrm{Mo}_{15} \mathrm{Se}_{19}$. Electron diffraction performed below and above $T_{\mathrm{CDW}}=114 \mathrm{~K}$ evidenced a weak commensurate modulation along the $c$ axis of the crystal structure which is observable neither by laboratory PXRD nor by single-crystal XRD. The characterization of the superconducting state that develops below $T_{\mathrm{c}}=2.8 \mathrm{~K}$ indicates that this compound can be classified as a weaklyto-intermediate-coupled type-II superconductor. Electronic band structure calculations suggest that $\mathrm{K}_{2} \mathrm{Mo15}_{15} \mathrm{Se}_{19}$ provides another example of CDW system where the transition is probably not driven by Fermi surface nesting but is more likely due to the $q$-dependence of the electronphonon coupling. The similarities between the transport properties measured in $\mathrm{K}_{2} \mathrm{Mo}_{15} \mathrm{Se}_{19}$ and those observed in other $A_{2} \mathrm{Mo}_{15} \mathrm{Se}_{19}$ compounds strongly suggest that the coexistence of a CDW state and superconductivity may be commonly observed in this family of compounds. Future systematic studies will be important to better understand their interplay and ascertain the physical origin of the CDW transition. In this respect, the chemical tuning afforded by this family and the application of external pressure or introduction of weak structural disorder will be important tools for tipping the balance between CDW and superconductivity in these compounds. 


\section{Acknowledgements}

C. C. acknowledge the financial support of the Pôle M4 of the University of Lorraine. R. A. R. A. O. acknowledges the Région Bretagne for a $\mathrm{PhD}$ grant. ${ }^{3} \mathrm{He}$ specific heat measurements were performed in MGML (www.mgml.eu), which is supported within the program of Czech Research Infrastructures (project no. LM2018096). 


\section{Tables}

Table 1. Summary of Superconducting Parameters of $\mathrm{K}_{2} \mathrm{Mo}_{15} \mathrm{Se}_{19}$.

\begin{tabular}{cc} 
Parameter & $\mathrm{K}_{2} \mathrm{Mo}_{15} \mathrm{Se}_{19}$ \\
\hline$T_{c} H_{c 2}(0)$ & $2.8 \mathrm{~K}$ \\
$\mu_{0} H_{c 1}(0)$ & $12 \mathrm{mT}$ \\
$\mu_{0} H_{c}(0)$ & $106 \mathrm{mT}$ \\
$\xi_{\mathrm{GL}}$ & $110 \AA$ \\
$\lambda_{\mathrm{GL}}$ & $2000 \AA$ \\
$\kappa_{G L}$ & 18 \\
$\gamma$ & $63 \mathrm{~mJ} \mathrm{~mol}^{-1} \mathrm{~K}^{-2}$ \\
$\Delta(0)$ & $0.40 \mathrm{meV}^{2}$ \\
$2 \Delta(0) / k_{B} T_{c}$ & 3.64 \\
$\lambda_{e-p h}$ & 0.61 \\
$\theta_{D}$ & $183 \mathrm{~K}$ \\
\hline
\end{tabular}


Table 2. Superconducting temperature $T_{\mathrm{c}}$ and possible charge-density-wave temperature $T_{\mathrm{CDW}}$ of the $A_{2} \mathrm{Mo}_{15} \mathrm{Se}_{19}(A=\mathrm{K}, \mathrm{Rb}, \mathrm{Tl}$ and $\mathrm{In})$ compounds reported herein (see Figure $\mathrm{S} 7$ in Supplemental Material [61]) and gathered in Ref. $58(A=\mathrm{Li}, \mathrm{Na}, \mathrm{Sn}$ and $\mathrm{Pb})$. No transport property measurements have been reported to date for these four last compounds. The symbol / corresponds to properties that have not yet been measured.

\begin{tabular}{l|l|l} 
Compound & $T_{\mathrm{c}}(\mathrm{K})$ & $T_{\mathrm{CDW}}(\mathrm{K})$ \\
\hline \hline $\mathrm{K}_{2} \mathrm{Mo}_{15} \mathrm{Se}_{19}$ & 2.8 & 114 \\
$\mathrm{Rb}_{2} \mathrm{Mo}_{15} \mathrm{Se}_{19}$ & $/$ & 125 \\
$\mathrm{In}_{2} \mathrm{Mo}_{15} \mathrm{Se}_{19}$ & 1.4 & 140 \\
$\mathrm{Tl}_{2} \mathrm{Mo}_{15} \mathrm{Se}_{19}$ & $<2$ & 140 \\
\hline \hline $\mathrm{Li}_{2} \mathrm{Mo}_{15} \mathrm{Se}_{19}$ & 2.6 & $/$ \\
$\mathrm{Na}_{2} \mathrm{Mo}_{15} \mathrm{Se}_{19}$ & $<0.5$ & $/$ \\
$\mathrm{Sn}_{2} \mathrm{Mo}_{15} \mathrm{Se}_{19}$ & $<0.5$ & $/$ \\
$\mathrm{Pb}_{2} \mathrm{Mo}_{15} \mathrm{Se}_{19}$ & $<0.5$ & $/$ \\
\hline \hline
\end{tabular}




\section{References}

[1] J. A. Wilson, F. J. Di Salvo, and S. Mahajan, Adv. Phys. 24, 117 (1975).

[2] P. Cottingham, D. C. Miller, J. P. Sheckelton, J. R. Neilson, M. Feygenson, A. Huq, and T. M. J. McQueen, Mater. Chem. C 2, 3238 (2014).

[3] G. Gruner in Density Waves in Solids (Addison-Wesley, Reading MA 1994).

[4] A. M. Gabovich, A. I. Voitenko, and M. Ausloos, Phys. Rep. 367, 583 (2002).

[5] F. J. Di Salvo, D. Moncton, and J. Waszczak, Phys. Rev. B 14, 4321 (1976).

[6] A. F. Kusmartseva, B. Sipos, H. Berger, L. Forró, and E. Tutis, Phys. Rev. Lett. 103, 236401 (2009).

[7] E. Morosan, H. W. Zandbergen, B. S. Dennis, J. W. G. Bos, Y. Onose, T. Klimczuk, A. P. Ramirez, N. P. Ong, and R. J. Cava, Nature Phys. 2, 544 (2006).

[8] H. Barath, M. Kim, J. F. Karpus, S. L. Cooper, P. Abbamonte, E. Fradkin, E. Morosan, and R. J. Cava, Phys. Rev. Lett. 100, 106402 (2008).

[9] C. S. Snow, J. F. Karpus, S. L. Cooper, T. E. Kidd, and T.-C. Chiang, Phys. Rev. Lett. 91, $136402(2003)$.

[10] T. Gruner, D. Jang, Z. Huesges, R. Cardoso-Gil, G. H. Fecher, M. M. Koza, O. Stockert, A. P. Mackenzie, M. Brando, and C. Geibel, Nat. Phys. 13, 967 (2017).

[11] L. E. Klintberg, S. K. Goh, P. L. Alireza, P. J. Saines, D. A. Tompsett, P. W. Logg, J. Yang, B. Chen, K. Yoshimura, and F. Malte Grosche, Phys. Rev. Lett. 109, 237008 (2012).

[12] X. Zhu, W. Ning, L. Li, L. Ling, R. Zhang, J. Zhang, K. Wang, Y. Liu, L. Pi, Y. Ma, H. Du, M. Tian, Y. Sun, C. Petrovic, and Y. Zhang, Sci. Rep. 6, 26974 (2016).

[13] W. C. Yu, Y. W. Cheung, P. J. Saines, M. Imai, T. Matsumoto, C. Michioka, K. Yoshimura, and S. K. Goh, Phys. Rev. Lett. 115, 207003 (2015). 
[14] T. Wu, H. Mayaffre, S. Kramer, M. Horvatic, C. Berthier, W. N. Hardy, R. Liang, D. A. Bonn, and M.-H. Julien, Nature 477, 191 (2011).

[15] G. Ghiringhelli, M. Le Tacon, M. Minola, S. Blanco-Canosa, C. Mazzoli, N. B. Brookes, G. M. De Luca, A. Frano, D. G. Hawthorn, F. He, T. Loew, M. Moretti Sala, D. C. Peets, M. Salluzzo, E. Schierle, R. Sutarto, G. A. Sawatzky, E. Weschke, B. Keimer, and L. Braicovich, Science 337, 821 (2012).

[16] I. M. Vishik, M. Hashimoto, R.-H. He, W.-S. Lee, F. Schmitt, D. Lu, R. G. Moore, C. Zhang, W. Meesavana, T. Sasagawa, S. Uchida, K. Fujita, S. Ishida, M. Ishikado, Y. Yoshida, H. Eisaki, Z. Hussain, T. P. Devereaux, and Z.-X. Shen, Proc. Natl. Acad. Sci. USA 109, 18332 (2012).

[17] J. Chang, E. Blackburn, A. T. Holmes, N. B. Christensen, J. Larsen, J. Mesot, R. Liang, D. A. Bonn, W. N. Hardy, A. Watenphul, M. V. Zimmermann, E. M. Forgan, and S. M. Hayden, Nat. Phys. 8, 871 (2012).

[18] M. Le Tacon, A. Bosak, S. M. Souliou, G. Dellea, T. Loew, R. Reid, K.-P. Bohnen, G. Ghiringhelli, M. Krisch, and B. Keimer, Nat. Phys. 10, 52 (2014).

[19] S. Nagata, T. Aochi, T. Abe, S. Ebisu, T. Hagino, Y. Seki, and K. Tsutsumi, J. Phys. Chem. Solids 53, 1259 (1992).

[20] T. Kumakura, H. Tan, T. Handa, M. Morishita, and H. Fukuyama, Czech. J. Phys. 46, 2611 (1996).

[21] M. Nunezregueiro, J. M. Mignot, M. Jaime, D. Castello, and P. Monceau, Synth. Met. 56, 2653 (1993).

[22] B. Mihaila, C. P. Opeil, F. R. Drymiotis, J. L. Smith, J. C. Cooley, M. E. Manley, A. Migliori, C. Mielke, T. Lookman, A. Saxena, A. R. Bishop, K. B. Blagoev, D. J. Thoma, J. C. Lashley, B. E. Lang, J. Boerio-Goates, B. F. Woodfield, and G. M. Schmiedeshoff, Phys. Rev. Lett. 96, 76401 (2006). 
[23] D. Jaiswal, A. A. Tulapurkar, S. Ramakrishnan, A. K. Grover, G. J. Nieuwenhuys, and J. Mydosh, Physica B 312, 142 (2002).

[24] Y. Singh, R. Nirmala, S. Ramakrishnan, and S. K. Malik, Phys. Rev. B 72, 45106 (2005). [25] Y. Nagano, N. Araoka, A. Mitsuda, H. Yayama, H. Wada, M. Ichihara, M. Isobe, and Y. Ueda, J. Phys. Soc. Jpn. 82, 064715 (2013).

[26] H. Luo, W. Xie, J. Tao, H. Inoue, A. Gyenis, J. W. Krizan, A. Yazdani, Y. Zhu, and R. J. Cava, Proc. Natl. Acad. Sci. USA 112, 1174 (2015).

[27] N. Yamamoto, R. Kondo, H. Maeda, and Y. Nogami, J. Phys. Soc. Jpn. 82, 123701 (2013). [28] M. Roman, J. Strychalska-Nowak, T. Klimczuk, and K. K. Kolincio, Phys. Rev. B 97, 041103(R) (2018).

[29] S. Steiner, H. Michor, O. Sologub, B. Hinterleitner, F. Höfenstock, M. Waas, E. Bauer, B. Stöger, V. Babizhetskyy, V. Levytskyy, and B. Kotur, Phys. Rev. B 97, 205115 (2018).

[30] M. Roman, L. Litzbarski, T. Klimczuk and K. K. Kolincio, Phys. Rev. B 99, 245152 (2019).

[31] J. Yang, B. Chen, C. Michioka, and K. Yoshimura, J. Phys. Soc. Jpn. 79, 113705 (2010).

[32] P. K. Biswas, A. Amato, R. Khasanov, H. Luetkens, K. Wang, C. Petrovic, R. M. Cook, M. R. Lees, and E. Morenzoni, Phys. Rev. B 90, 144505 (2014).

[33] C. N. Kuo, W. T. Chen, C. W. Tseng, C. J. Hsu, R. Y. Huang, F. C. Chou, Y. K. Kuo and C. S. Lue, Phys. Rev. B 97, 094101 (2018).

[34] K. Cho, M. Konczykowski, S. Teknowijoyo, M. A. Tanatar, J. Guss, P. B. Gartin, J. M. Wilde, A. Kreyssig, R. J. McQueeney, A. I. Goldman, V. Mishra, P. J. Hirschfeld and R. Prozorov, Nature Comm. 9, 2796 (2018).

[35] L. Li, X. Deng, Z. Wang, Y. Liu, M. Abeykoon, E. Dooryhee, A. Tomic, Y. Huang, J. B. Warren, E. S. Bozin, S. J. L. Billinge, Y. Sun, Y. Zhu, G. Kotliar and C. Petrovic, npj Quantum Mater. 2, 11 (2017). 
[36] R. Shimano, and N. Tsuji, Annu. Rev. Condens. Matter Phys. 11, 103 (2020).

[37] R. Grasset, T. Cea, Y. Gallais, M. Cazayous, A. Sacuto, L. Cario, L. Benfatto, and M.-A. Méasson, Phys. Rev. B 97, 094502 (2018).

[38] T. Cea, and L. Benfatto, Phys. Rev. B 90, 224515 (2014).

[39] M.-A. Méasson, Y. Gallais, M. Cazayous, B. Clair, P. Rodière, L. Cario, and A. Sacuto, Phys. Rev. B 89, 060503 (2014).

[40] T. Zhou, B. Lenoir, M. Colin, A. Dauscher, R. Al Rahal Al Orabi, P. Gougeon, M. Potel, and E. Guilmeau, Appl. Phys. Lett. 98, 162106 (2011).

[41] T. Zhou, M. Colin, C. Candolfi, C. Boulanger, A. Dauscher, E. Santava, J. Hejtmanek, P. Baranek, R. Al Rahal Al Orabi, M. Potel, B. Fontaine, P. Gougeon, R. Gautier, and B. Lenoir, Chem. Mater. 26, 4765 (2014).

[42] R. Al Rahal Al Orabi, P. Gougeon, P. Gall, B. Fontaine, R. Gautier, M. Colin, C. Candolfi, A. Dauscher, J. Hejtmanek, B. Malaman, and B. Lenoir, Inorg. Chem. 53, 11699 (2014).

[43] P. Gougeon, P. Gall, R. Al Rahal Al Orabi, B. Fontaine, R. Gautier, M. Potel, T. Zhou, B. Lenoir, M. Colin, C. Candolfi, and A. Dauscher, Chem. Mater. 24, 2899 (2012).

[44] R. Al Rahal Al Orabi, B. Fontaine, R. Gautier, P. Gougeon, P. Gall, Y. Bouyrie, A. Dauscher, C. Candolfi, and B. Lenoir, Inorg. Chem. 55, 6616 (2016).

[45] G. Daigre, P. Gougeon, P. Gall, R. Gautier, O. Guillou, J.-B. Vaney, C. Candolfi, A. Dauscher, and B. Lenoir, J. Solid State Chem. 237, 1 (2016).

[46] P. Masschelein, C. Candolfi, A. Dauscher, C. Gendarme, R. Al Rahal Al Orabi, P. Gougeon, M. Potel, P. Gall, R. Gautier, and B. Lenoir, J. Alloys Compnd 739, 360 (2018).

[47] M. Colin, T. Zhou, B. Lenoir, A. Dauscher, R. Al Rahal Al Orabi, P. Gougeon, M. Potel, P. Baranek, and C. Semprimoschnig, J. Electron. Mater. 41, 1360 (2012).

[48] P. Gougeon, P. Gall, O. Merdrignac-Conanec, L. Aranda, A. Dauscher, C. Candolfi, and B. Lenoir, Inorg. Chem. 56, 9684 (2017). 
[49] P. Gougeon, P. Gall, R. Al Rahal Al Orabi, B. Boucher, B. Fontaine, A. Dauscher, C. Candolfi, and B. Lenoir, Inorg. Chem. 58, 5533 (2019).

[50] R. Al Rahal Al Orabi, B. Boucher, B. Fontaine, P. Gall, C. Candolfi, B. Lenoir, P. Gougeon, J.-F. Halet, and R. Gautier, J. Mater. Chem. C 5, 12097 (2017).

[51] P. Gougeon, P. Gall, R. Gautier, and M. Potel, Acta Crystallogr. C66, i67 (2010).

[52] P. Gougeon, M. Potel, J. Padiou, and M. Sergent, Mater. Res. Bull. 22, 1087 (1987).

[53] S. Picard, P. Gougeon, and M. Potel, Angew. Chem. 38, 2034 (1999).

[54] M. Giroud, J.-L. Genicon, R.Tournier, C. Geantet, O. Peña, R. Horyn, and M. Sergent, J. Low Temp. Phys. 69, 419 (1987).

[55] W. Thomlinson, G. Shirane, D. E. Moncton, M. Ishikawa, and Ø. Fischer, Phys. Rev. B 23, 4455 (1981)

[56] M. Ishikawa, and Ø. Fischer, Solid State Commun. 23, 37 (1977).

[57] M. Ishikawa, and Ø. Fischer, Solid State Commun. 24, 747 (1977).

[58] C. K. Poole, H. A. Farach, and R. J. Creswick, in Handbook of Superconductivity, Academic Press (2000).

[59] R. Chevrel, M. Potel, and M. Sergent, Mat. Res. Bull. 15, 867 (1980).

[60] L. J. Farrugia, J. Appl. Cryst. 45, 849 (2012).

[61] See Supplemental Material for additional electron diffraction images, crystallographic details inferred from single-crystal X-ray diffraction, additional images of the electronic band structure and a comparison of the temperature dependence of the electrical resistivity of the $A_{2} \mathrm{Mo}_{15} \mathrm{Se}_{19}$ compounds $(A=\mathrm{K}, \mathrm{Rb}, \mathrm{In}$ and $\mathrm{Tl})$.

[62] R. Prozorov, and V. G. Kogan, Phys. Rev. Appl. 10, 014030 (2018).

[63] P. Blaha, K. Schwarz, G. K. H. Madsen, D. Kvasnicka, and J. Luitz, WIEN2k 14.2, Vienna, Austria (2011). 
[64] J. P. Perdew, K. Burke, and M. Ernzerhof, Phys. Rev. Lett. 77, 3865 (1996).

[65] A. Kokalj, Comp. Mater. Sci. 28, 155 (2003).

[66] B. Becker, N. G. Patil, S. Ramakrishnan, A. A. Menovsky, G. J. Nieuwenhuys, J. A. Mydosh, M. Kohgi, and K. Iwasa, K. Phys. Rev. B 59, 7266 (1999).

[67] Y.-K. Kuo, F. H. Hsu, H. H. Li, H. L. Huang, C. W. Huang, C. S. Lue, and H. D. Yang, Phys. Rev. B 67, 195101 (2003).

[68] J. D. Jorgensen, and D. G. Hinks, Physica B 136B, 485 (1986).

[69] M. François, K. Yvon, D. Cattani, M. Decroux, R. Chevrel, M. Sergent, S. Boudjada, and Th. Wroblewski, J. Appl. Phys. 75, 423 (1994).

[70] K. Yvon, R. Baillif, and R. Flükiger, Acta Cryst. 35, 2859 (1979).

[71] K. Yvon, R. Chevrel, and M. Sergent, Acta Cryst. 36, 685 (1980).

[72] F. Kubel, and K. Yvon, Acta Cryst. 46, 181 (1990).

[73] B. Koppelhuber-Bitschnau, F. A. Mautner, and K. Yvon, Monatsh. Chem. 121, 505 (1990).

[74] J. M. Tarascon, F. J. DiSalvo, D. W. Murphy, G. Hull, and J. V. Waszczak, Phys. Rev. B 29, 172 (1984).

[75] V. F. Shamrai, G. M. Leitus, and S. G. Zhukov, Kristallografiya 35, 349 (1990).

[76] W. L. McMillan, Phys. Rev. 167, 331 (1968).

[77] R. E. Peierls, in Quantum Theory of Solids (Oxford University Press, London, p. 108, 1955).

[78] A. Seidel, C. Marianetti, F. C. Chou, G. Ceder, and P. Lee, Phys. Rev. B 67, 020405 (2003).

[79] M. D. Johannes, and I. I. Mazin, Phys. Rev. B 77, 165135 (2008).

[80] L. P. Gor'kov, Phys. Rev. B 85, 165142 (2012).

[81] S. Steiner, H. Michor, O. Sologub, B. Hinterleitner, F. Höfenstock, M. Waas, E. Bauer, B. Stöger, V. Babizhetskyy, V. Levytskyy, and B. Kotur, Phys. Rev. B 97, 205115 (2018). 
[82] X. Zhu, Y. Cao, J. Zhang, E. W. Plummer, and J. Guo, Proc. Natl. Acad. Sci. USA 112, 2367 (2015).

[83] D. Shoenberg, in Magnetic Oscillations in Metals (Cambridge University Press, Cambridge, 1984).

[84] D. J. Rebar, S. M. Birnbaum, J. Singleton, M. Khan, J. C. Ball, P. W. Adams, J. Y. Chan, D. P. Young, D. A. Browne, and J. F. DiTusa, Phys. Rev. B 99, 094517 (2019).

[85] K. Ohgushi, A. Yamamoto, Y. Kiuchi, C. Ganguli, K. Matsubayashi, Y. Uwatoko, and H. Takagi, Phys. Rev. Lett. 106, 017001 (2011).

[86] M. J. Winiarski, B. Wiendlocha, M. Sternik, P. Wisniewski, and J. R. O’Brien, Phys. Rev. B 93, 134507 (2016).

[87] Z. Hiroi, A. Onosaka, Y. Okamoto, J.-Y. Yamaura, and H. J. Harima, Phys. Soc. Jpn. 81, 124707 (2012).

[88] T. Shimojima, Y. Shibata, K. Ishizaka, T. Kiss, A. Chainani, T. Yokoya, T. Togashi, X.Y. Wang, C. T. Chen, S. Watanabe, J. Yamaura, S. Yonezawa, Y. Muraoka, Z. Hiroi, S. Saitoh, and S. Shin, Phys. Rev. Lett. 99, 117003 (2007). 


\section{Figure Captions}

Figure 1. (left panel) View of the hexagonal crystal structure of $\mathrm{K}_{2} \mathrm{Mo}_{15} \mathrm{Se}_{19}$ (space group $R \overline{3} c$, No. $167, Z=6, a=9.7093 \AA$ and $c=58.1385 \AA$ at $300 \mathrm{~K}$ ) along the $a$ axis showing the spatial arrangement of the $\mathrm{Mog}_{9} \mathrm{Se}_{11}$ clusters interconnected by smaller $\mathrm{Mo6}_{6} \mathrm{Se}$ clusters. The $\mathrm{K}$ atoms are shown in light blue. The structure refinement details and crystallographic parameters determined by single-crystal X-ray diffraction at $300 \mathrm{~K}$ are given in Tables $\mathrm{S} 1$ and $\mathrm{S} 2$, respectively, in Supplemental Material [61]. (right panel) Crystal structure projected along the $c$ axis. In both panels, the hexagonal unit cell is represented by the black solid lines.

Figure 2. Experimental signatures of the charge density wave order in $\mathrm{K}_{2} \mathrm{Mo}_{15} \mathrm{Se}_{19}$ observed in the temperature dependence of the a) electrical resistivity $\rho, \mathrm{b})$ magnetization $M, \mathrm{c})$ specific heat $C_{p}$ and d) thermopower $\alpha$. The crossing solid black lines show how the transition temperature $T_{\mathrm{CDW}}$ was determined. In panel $\mathrm{b}$ ), the small hump seen around $50 \mathrm{~K}$ is presumably due to a small concentration of oxygen trapped in the measurement system.

Figure 3. a) HRTEM image obtained on single-crystalline $\mathrm{K}_{2} \mathrm{Mo}_{15} \mathrm{Se}_{19}$ taken along the [10 $\left.\overline{1} 0\right]$ zone axis at $300 \mathrm{~K}$. Inset: electron diffraction pattern of the HRTEM image. b) HAADF-STEM image taken along the [10 10$]$ zone axis at $300 \mathrm{~K}$. The sublattice of Mo atoms, shown in dark blue, is overlaid. The inset shows the inverse Fast-Fourier transform (IFFT) of the HAADFSTEM image. Electron diffraction patterns taken along the [10̄0] zone axis c) at $300 \mathrm{~K}$ and d) below the transition temperature $T_{\mathrm{CDW}}=114 \mathrm{~K}$. The additional Bragg reflections due to the structural modulation below $T_{\mathrm{CDW}}$ are clearly observed along $c^{*}$, as shown by the vertical black arrows. 
Figure 4. Experimental signatures of bulk superconductivity in $\mathrm{K}_{2} \mathrm{Mo}_{15} \mathrm{Se}_{19}$ observed in the temperature dependence of a) the electrical resistivity $\rho, \mathrm{b}$ ) the specific heat $C_{p}$ and c) the magnetic susceptibility $\chi$. The drop in $\rho$ and $\chi$ combined with the lambda-type anomaly in $C_{p}$ demonstrates that bulk superconductivity develops below $T_{\mathrm{c}}=2.8 \mathrm{~K}$. The superconducting transition temperature $T_{\mathrm{c}}$ was determined as the endpoint of the resistivity transition, that is, when $\rho$ reaches zero. The specific heat data were measured down to $0.35 \mathrm{~K}$ and under applied magnetic fields of 1T, 2T and 3T. The zero-field cooled (ZFC) temperature-dependent volume magnetic susceptibility $\chi$ was measured under an applied field of 10 Oe. The data were corrected for a demagnetization factor $N=0.456$ determined from the shape and geometry of the sample measured and the formula derived for a cuboid-shaped sample in Ref. 62.

Figure 5. (a) Magnification around the Fermi level $E_{F}$ of the electronic dispersion curves of $\mathrm{K}_{2} \mathrm{Mo}_{15} \mathrm{Se}_{19}$, calculated along high-symmetry directions in the Brillouin zone. In these calculations, spin-orbit coupling was not taken into account. The four bands crossing $E_{F}$ are shown as solid blue and orange curves and dotted black and green curves. (b) Perspective views and projection along the $c^{*}$ axis of the Fermi surface sheets of $\mathrm{K}_{2} \mathrm{Mo}_{15} \mathrm{Se}_{19}$, calculated without spin-orbit coupling, shown separately for the four bands crossed by the Fermi level. The Fermi surface comprises hole-like cylinders centered at the zone center (two first top panels), small electron-like pockets (middle panel) and an electron-like "monster" (before-last panel). The total Fermi surface is shown in the last bottom panel. 




Figure 1 

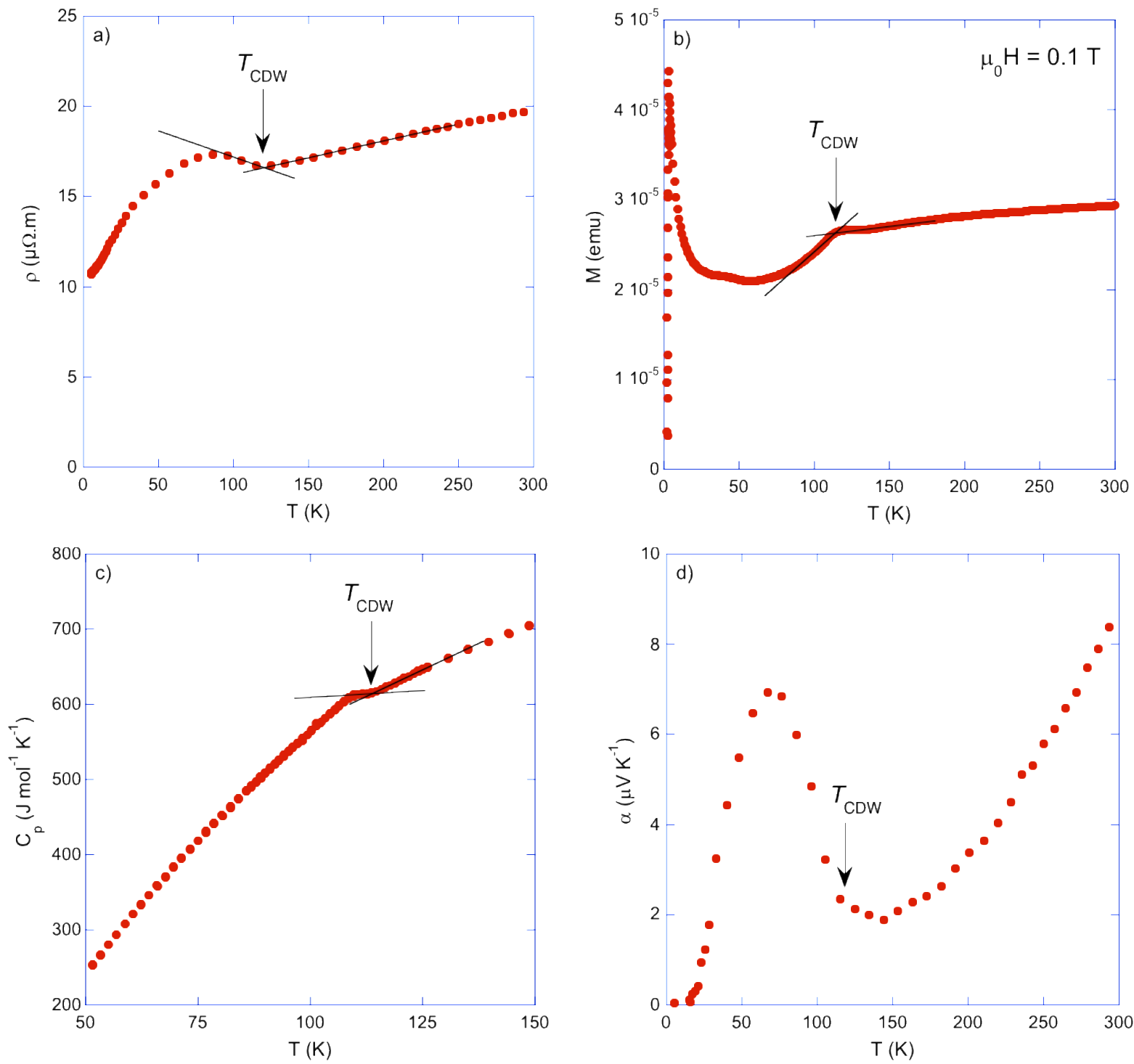

Figure 2 
a)

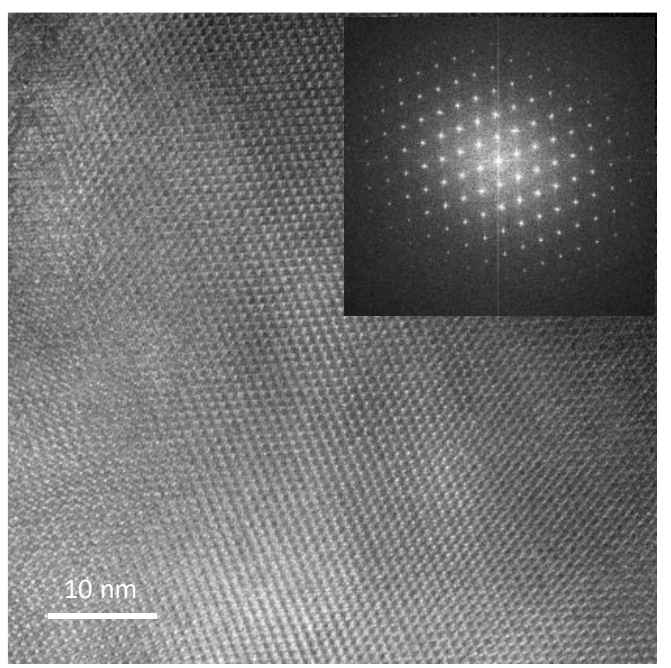

c)

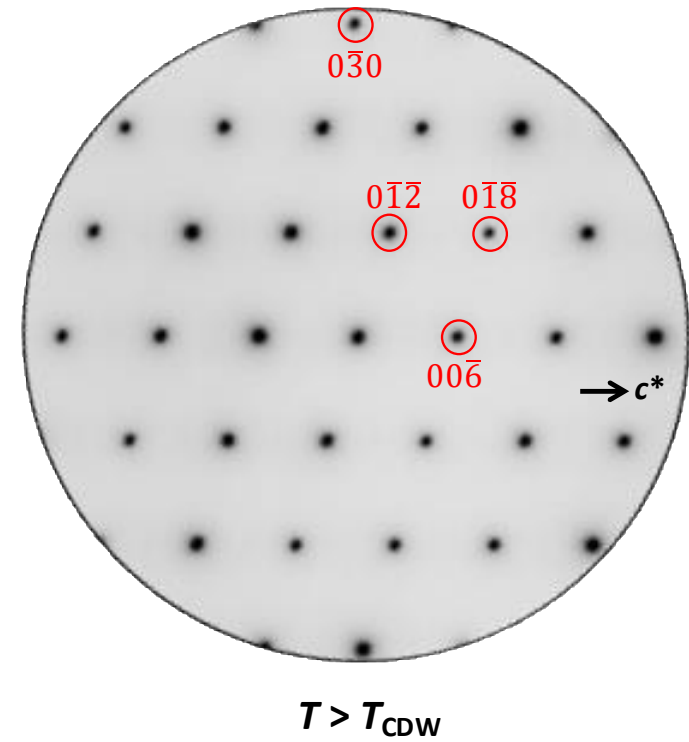

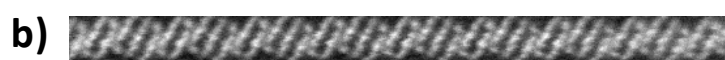

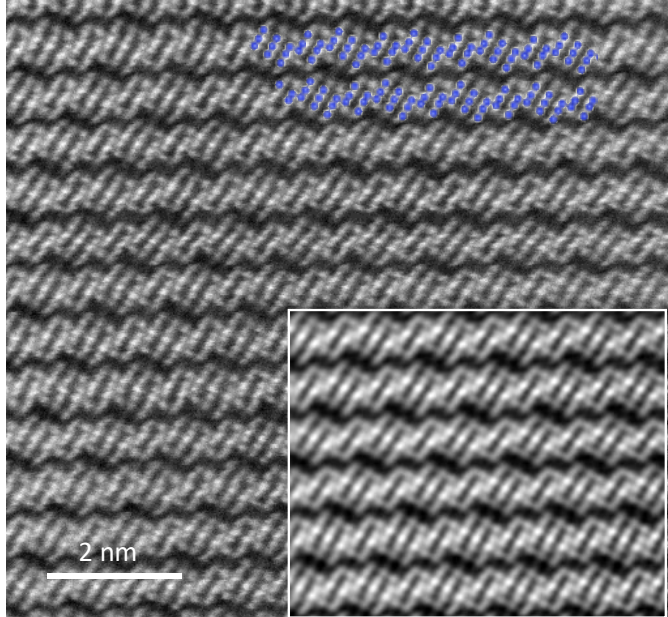

d)

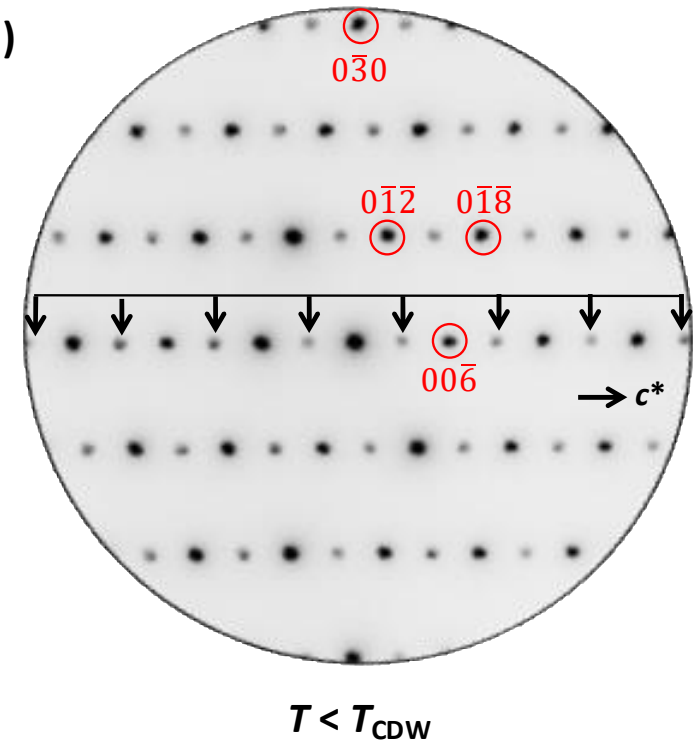

Figure 3 



Figure 4 
(a)

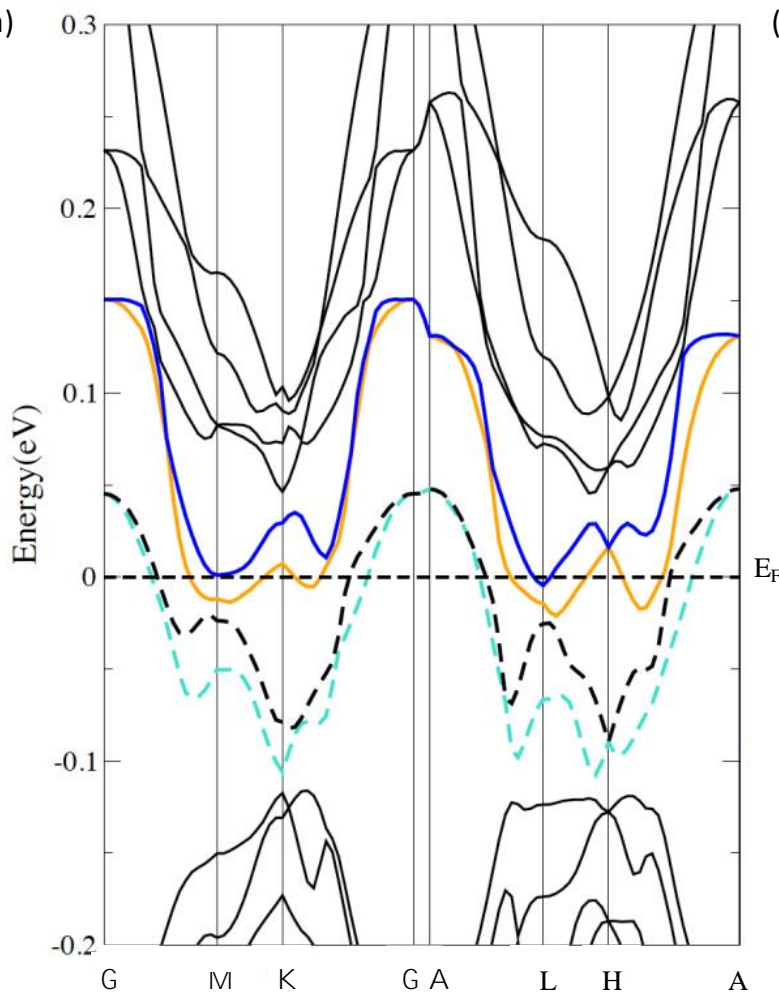

(b)
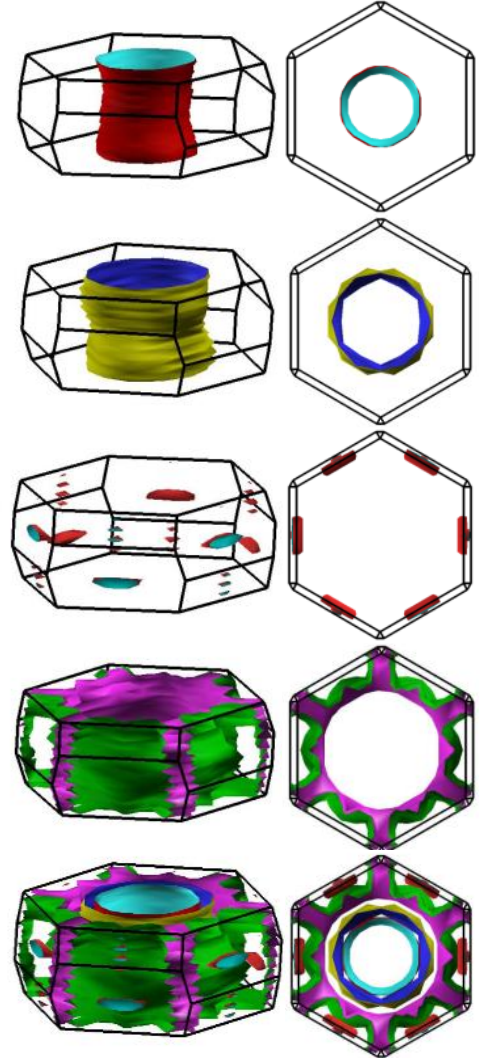

Figure 5 